\title{
ON THE KERNEL OF A MARKOV PROJECTION ON $C(X)$
}

\author{
ROBERT E. ATALLA
}

\begin{abstract}
Let $X$ be a compact metric space and $L$ a closed linear subspace of $C(X)$, the real valued continuous functions on $X$. We give necessary and sufficient conditions of an algebraic nature for $L$ to be the kernel of a Markov projection $P$ on $C(X)$. We also characterize compact spaces for which our result holds as those for which the Borsuk-Dugundji simultaneous extension theorem holds.
\end{abstract}

1. Introduction. A projection $P$ on $C(X)$ is Markov if $P e=e$ (where $e$ is the unit function) and $P \geqslant 0$, i.e., $f \geqslant 0$ implies $P f \geqslant 0$. If $P^{*}$ is the adjoint of $P$ and $\delta_{x}$ the Dirac measure at $x$, let $p_{x}=P^{*} \delta_{x}$, so that $p_{x}$ is a probability measure, and for $f \in C(X)$ we have $P f(x)=\int f d p_{x}$. Let $\mathbf{P}$ be the set of Borel probability measures on $X$, a compact convex set in $C(X)^{*}$, relative to the weak*-topology. Then $P^{*}(\mathbf{P})$ is a compact convex set, and each extreme point $m$ has the form $p_{x}$ for some $x \in X$-just note that $p^{*-1}(m)$ is a convex compact subset of $\mathbf{P}$, and hence contains an extreme point, which is a $\delta_{x}$ for some $x \in X[4$, p. 34].

If $m$ is a positive Borel measure, supp $m$ denotes the closed support set of $m$, and if $m$ is any Borel measure, supp $m$ is defined as supp $|m|$. If $P$ is a Markov projection, we define $\operatorname{supp} P=\operatorname{closure} \bigcup\left\{\operatorname{supp} m: P^{*} m=m\right\}$. (Note that $m \in \operatorname{ran} P^{*}$ iff $P^{*} m$ $=m$.)

The structure of $P$ is pretty well known. Birkhoff [1] and Kelley [3] characterized those $P$ for which $\operatorname{ran} P$ is an algebra by the following properties: for each $x \in X, p_{x}$ is an extreme point of $P^{*}(\mathbf{P})$, and for each $f \in C(X), P f$ is constant on supp $p_{x}$. Moreover, $P$ satisfies the averaging identity $P(f P g)=P f P g$. Lloyd [5] showed that if $P$ is an arbitrary Markov projection, then $P f$ is constant on supp $p_{x}$ whenever $p_{x}$ is an extreme point of $P^{*}(\mathbf{P})$. It follows easily that the natural restriction of $P$ to a projection on $C(\operatorname{supp} P)$ satisfies the Birkhoff-Kelley conditions. Later Lloyd and Seever found the following identity for all Markov projection: $P(f P g)=P(P f P g)$ ([6 and 7], see also [9]).

This formula may be rewritten as $0=P((f-P f) P g)$, i.e., if $f_{0} \in \operatorname{ker} P$ and $g_{0} \in \operatorname{ran} P$, then $f_{0} g_{0} \in \operatorname{ker} P$. This condition is not quite strong enough to characterize the kernel of a Markov projection, so we note a natural property of such projections, namely if $f \geqslant 0$, then $P f=0$ iff $f$ vanishes on supp $P$. This is an obvious consequence of the fact that for $x \in X, p_{x}$ is a probability measure. Thus, if $P$ is a

Received by the editors April 24, 1984.

1980 Mathematics Subject Classification. Primary 47A65, 47B55.

Key words and phrases. Markov projection, $C(X)$, averaging operator, positive Borel measure.

(c1985 American Mathematical Society $0002-9939 / 85 \$ 1.00+\$ .25$ per page 
Markov projection we have

(1) ker $P+\operatorname{ran} P=C(X)$,

(2) $($ ran $P)(\operatorname{ker} P) \subset \operatorname{ker} P$,

(3) $I=\left\{f: P f^{2}=0\right\}$ is an ideal in $C(X)$.

(Note that if $m$ is a nonpositive Borel measure with $m(e)=1$, and we define $P$ by $P f(x)=m(f)$ for all $f \in C(X)$, then (1) and (2) hold, but not (3).)

Our main result is

ThEOREM. Let $X$ be compact metric, $L$ a proper closed linear subspace of $C(X)$, and $M=\{f: f L \subset L\}$. If

(a) $L+M=C(X)$, and

(b) $I=\left\{f: f^{2} \in L\right\}$ is an ideal,

then there exists a Markov projection $P$ on $C(X)$ such that $L=\operatorname{ker} P$ and $\operatorname{ran} P \subset M$.

2. Preliminaries. Throughout, $L$ will be a closed subspace of $C(X)$, the real valued continuous functions on $X$, and $M$ and $I$ are as defined in the Theorem. In this section we study the structure of $I$ after we give some definitions.

Let $L^{\perp}=\left\{m \in C(X)^{*}: m(f)=0\right.$ for all $\left.f \in L\right\}$, and let $\left(L^{\perp}\right)_{1}$ be the closed unit ball in $L^{\perp}$, a compact convex set in the weak*-topology. Note that $f \in L$ iff $m(f)=0$ for all $m \in L^{\perp}$ (by Hahn-Banach). Obviously, $f \in M$ iff $f d m \in L^{\perp}$ for all $m \in L^{\perp}$, so $M=\left\{f \in C(X): f L^{\perp} \subset L^{\perp}\right\}$. Further, $f \in M$ iff $f$ is constant on supp $m$ for each extreme point $m \in\left(L^{\perp}\right)_{1}$ [4, pp. 35-36]. We also define $Z(I)$ $=\left\{f^{-1}(0): f \in I\right\}$ and $\operatorname{supp} L^{\perp}=\operatorname{closure} \bigcup\left\{\operatorname{supp} m: m \in L^{\perp}\right\}$. If $f \in C(X)$ and $A \subset X$, then $f_{A}$ is the restriction of $f$ to $A$, and $L_{A}=\left\{f_{A}: f \in L\right\}$.

2.1 Remark. $Z(I) \subset \operatorname{supp} L^{\perp}$.

Proof. If $x \notin \operatorname{supp} L^{\perp}$, then by complete regularity there exists $f \in C(X)$ which vanishes on $\operatorname{supp} L^{\perp}$, but $f(x) \neq 0$. Then $f^{2} \in L$, so $f \in I$ and $x \notin Z(I)$.

2.2 Proposition. The following are equivalent:

(a) $I$ is an ideal,

(b) $Z(I)=\operatorname{supp} L^{\perp}$.

Proof. (b) implies (a). We show $f \in I$ iff supp $L^{\perp} \subset f^{-1}(0)$, so that $I$ is the ideal $\left\{g: \operatorname{supp} L^{\perp} \subset g^{-1}(0)\right\}$. If $f \in I$, then (b) implies $\operatorname{supp} L^{\perp} \subset f^{-1}(0)$. If $\operatorname{supp} L^{\perp} \subset$ $f^{-1}(0)$, then for all $m \in L^{\perp}, 0=m\left(f^{2}\right)$, so $f^{2} \in L$ and $f \in I$.

(a) implies (b). To show supp $L^{\perp} \subset Z(I)$, let $f \in I$ and $m \in L^{\perp}$. Let $m=m^{+}-m^{-}$ be the Lebesgue decomposition with $m^{+}$supported by the Baire set $A$ and $m^{-}$ supported by $X \backslash A$. Let $g_{n} \in C(X)$ with $1 \geqslant g_{n} \geqslant 0$ and $g_{n} \rightarrow 1_{A}|m|$-a.e. Now $f g_{n} \in I$ so $f^{2} g_{n}^{2} \in L$, and

$$
\int f^{2} d m^{+}=\int f^{2} 1_{A} d m=\lim \int f^{2} g_{n}^{2} d m=0
$$

since $m \in L^{\perp}$. Likewise $\int f^{2} d m^{-}=0$, so $f^{2}=0|m|$-a.e. By continuity, supp $m \subset$ $f^{-1}(0)$, and since $m$ is arbitrary, supp $L^{\perp} \subset f^{-1}(0)$.

2.3 Proposition. If $M+L=C(X)$ and $m$ is an extreme point of $\left(L^{\perp}\right)_{1}$, then $m(e) \neq 0$. 
Proof. Let $S=\operatorname{supp} m$. (Since $L$ is proper, $m \neq 0$.) If $f \in M$ then $f$ is constant on $S$. By hypothesis $C(S)=L_{S}+M_{S}$. But then $C(S)=L_{S}+$ constants, so if $g \in C(S)$ we have $g=h+c e$ with $h \in L_{S}$ and constant, whence $m(g)=m(h)+c m(e)=0$ $+c m(e)$. If $m(e)=0$, then $m=0$, which is impossible.

2.4 Proposition. If $L+M=C(X)$, then (a) and (b) in 2.2 are equivalent to (c) $I \subset M$.

Proof. (b) implies (c). If $f \in I$, then $f$ is constant (in fact, 0 ) on supp $m$ whenever $m \in L^{\perp}$. Hence, $f \in M[4$, pp. 35-36].

(c) implies (b). By 2.1 we always have $Z(I) \subset \operatorname{supp} L^{\perp}$. Conversely, if $f \in I$, then (c) implies $f$ is constant on supp $m$ whenever $m$ is extreme in $\left(L^{\perp}\right)_{1}$. But since $f^{2} \in L$ as well, $m\left(f^{2}\right)=0$. Since $m(e) \neq 0, f^{2}$ must be 0 on supp $m$. It is an easy consequence of Krein-Milman that sets of the form supp $m$, with $m$ extreme in $\left(L^{\perp}\right)_{1}$, are dense in supp $L^{\perp}$, so supp $L^{\perp} \subset f^{-1}(0)$.

2.5 Proposition. Let $I_{0}=\left\{f \in C(X): f \in L\right.$ and $\left.f^{2} \in L\right\}$. If $I$ is an ideal, then $I=I_{0}$, and hence $I \subset L \cap M$, provided $L+M=C(X)$.

Proof. Clearly, $I_{0} \subset I$. If $I$ is an ideal, then $Z(I)=\operatorname{supp} L^{\perp}$, by 2.2 , so if $f \in I$, then $0=m(f)=m\left(f^{2}\right)$ for all $m \in L^{\perp}$, whence $f \in L$ as well as $f^{2} \in L$. Thus $f \in I_{0}$.

2.6 Remark. Propositions 2.2 and 2.4 remain true if $I$ is replaced by $I_{0}$. This fact is not needed below, and we omit the easy proof. In $\$ 4$ we give some examples on the relation between $I$ and $I_{0}$.

3. Proof of Theorem. (i) Let $Z=Z(I)$. By 2.4, hypotheses (a) and (b) of the Theorem imply $Z=\operatorname{supp} L^{\perp}$. We now prove $I=L \cap M$. By 2.5 we already have $I \subset L \cap M$. Conversely, if $f \in L \cap M$, then $f$ is constant on supp $m$ for $m$ extreme in $\left(L^{\perp}\right)_{1}$, while $m(f)=0$ because $f \in L$. Since by $2.3 m(e) \neq 0$, we have $f=0$ on supp $m$. It follows that $\operatorname{supp} L^{\perp} \subset f^{-1}(0)$, so $f \in I$.

(ii) Since $C(X)=L+M, I=L \cap M$, and $Z=Z(I)$, we have $C(Z)=L_{Z} \oplus M_{Z}$. Thus, there exists a projection $Q$ on $C(Z)$ whose kernel is $L_{Z}$ and whose range is $M_{Z}$. If $e_{Z}$ is the restriction of $e$ to $Z$, then clearly $Q e_{Z}=e_{Z}$, and it remains to show that $Q \geqslant 0$ (and then that $Q$ extends to a Markov projection $P$ on $C(X)$ ).

(iii) First we show that because (1) $\operatorname{ran}(Q) \operatorname{ker}(Q) \subset \operatorname{ker}(Q)$ and $(2) \operatorname{ran}(Q)$ is an algebra, we have $Q(f Q g)=Q f Q g$ for all $f$ and $g$ in $C(Z)$.

$$
\begin{aligned}
Q(f Q g) & =Q((f-Q f+Q f) Q g)=Q((f-Q f) Q g+Q(Q f Q g)) \\
& =0+Q f Q g .
\end{aligned}
$$

(iv) Secondly, if $f \geqslant 0$ and $Q f=0$, then $f=0$ on $Z$. Let $F \in C(X)$ satisfy $F \geqslant 0$ and $F_{Z}=f$. Since $f \in L_{Z}$, there exists $G \in L$ with $G_{Z}=f$, i.e., $G_{Z}=F_{Z}$. If $m \in L^{\perp}$, then supp $m \subset Z$, so $m(F)=m(G)=0$, so $F \in L$. Since $F \geqslant 0$, we have $F^{1 / 2} \in I \subset M$. Since $M$ is an algebra, $F \in M$, i.e., $F \in L \cap M=I$, so $f=F_{Z}=0$.

(v) Finally, suppose there exists $f \in C(Z)$ with $f \geqslant 0$, but $Q f(x)<0$ for some $x$. The set $V=\{y: Q f(y)<0\}$ is open in $Z$ relative to the topology generated by the 
subalgebra $M_{Z}=Q(C(Z))$, which is completely regular, but not Hausdorff. Hence, there exists $g \in M_{Z}$ such that $g(x)=1, g=0$ off $V$, and $0 \leqslant g \leqslant 1$. Let $h=g f$. Then $h \geqslant 0$, and, by (iii), $Q h=Q(g f)=Q((Q g) f)=Q g Q f=g Q f$. So $Q h(x)=$ $Q f(x)<0, Q h \leqslant 0$ on $V$, and $Q h=0$ off $V$. Let $k=h-Q h \geqslant 0$. Then $Q k=0$, so, by (iv), $k=0$ on $Z$, i.e., $h=Q h$. But this is impossible since $h(x) \geqslant 0$ and $Q h(x)<0$. (The last three lines were inspired by a homework paper of graduate student Pengyuan Chen.)

(vi) We now show that $Q$ extends to a Markov projection on $C(X)$. Since $X$ is compact metric (and this is the only time metrizabilty is used) there exists a simultaneous extender, i.e., a positive linear map $E: C(Z) \rightarrow C(X)$ such that, for $x \in Z, f(x)=E f(x)$, and also $E e_{Z}=e_{X}=e$. (See the Borsuk-Dugundji theorem in [8, p. 365].) We define $P$ by $P f(x)=E\left(Q\left(f_{Z}\right)\right)(x)$. It is easy to check that $P$ is a Markov projection, and we must show that $L=\operatorname{ker} P$ and $\operatorname{ran} P \subset M$.

(vii) To show $L \subset \operatorname{ker} P$, if $f \in L$, then $f_{Z} \in L_{Z}$, so $P f=E\left(Q\left(f_{Z}\right)\right)=E(0)=0$. To show ker $P \subset L$, suppose $0=P f=E\left(Q\left(f_{Z}\right)\right)$. If $m \in C(X)^{*}$ and supp $m \subset Z$, let $m_{Z}$ be $m$ considered as an element of $C(Z)^{*}$, so for $g \in C(X), m(g)=m_{Z}\left(g_{Z}\right)$, and for $g \in C(Z), m_{Z}(g)=m(E g)$. Then $m \in L^{\perp}$ iff $m_{Z} \in\left(L_{Z}\right)^{\perp}$. Since $L_{Z}=$ $\operatorname{ker} Q$ and $Q$ is a projection, $\left(L_{Z}\right)^{\perp}=\operatorname{ran}\left(Q^{*}\right)$, so $m \in L^{\perp}$ iff $Q^{*} m_{Z}=m_{Z}$. Hence, for all $m \in L^{\perp}$,

$$
\begin{aligned}
m(f) & =m_{Z}\left(f_{Z}\right)=Q^{*} m_{Z}\left(f_{Z}\right)=m_{Z}\left(Q\left(f_{Z}\right)\right)=m\left(E\left(Q f_{Z}\right)\right) \\
& =m(P f)=m(0)=0 .
\end{aligned}
$$

It follows that $f \in L$.

(viii) To show $\operatorname{ran} P \subset M$, since $L=\operatorname{ker} P$ and $P$ is a Markov operator, property (2) of the introduction says $(\operatorname{ran} P) L \subset L$.

4. Examples. We assumed metrizability of $X$ only in order to invoke the BorsukDugundji extension theorem. The following rather surprising result shows that the extension theorem is necessary as well as sufficient.

4. 1 Proposition. If $X$ is a compact Hausdorff space, the following are equivalent:

(a) If $Z$ is a closed subset, there exists a Markov extension operator $E: C(Z) \rightarrow$ $C(X)$.

(b) The result of our main theorem holds for $C(X)$.

Proof. We already know that (a) implies (b). Conversely, suppose (b) holds. If $Z$ is closed in $X$, let $L=\left\{f: f_{Z}=0\right\}$ be an ideal. Then $I=L$, so $I$ is an ideal, and $M=C(X)$, so $M+L=C(X)$. By (b) there exists a Markov projection $P$ with ker $P=L$. Now ran $P^{*}=L^{\perp}=C(Z)^{*}$, the space of regular Borel measures on $Z$. That is, if $m \in C(X)^{*}$ and supp $m \subset Z$, then $P^{*} m=m$. We define the extension operator $E$ as follows: if $f \in C(Z)$, let $f_{1}$ be any norm-preserving extension of $f$ to an element of $C(X)$, and let $E f=P f_{1}$. To show $E$ is well defined, suppose $f_{2}$ is any other extension of $f$ to an element of $C(X)$. If $x \in X$, then $\operatorname{supp} p_{x} \subset Z$, so $P f_{1}(x)=P f_{2}(x)=\int f d p_{x}$. To show $E$ is an extension operator, i.e., $(E f)_{Z}=f$, let $x \in Z$. Then $P^{*} \delta_{x}=\delta_{x}$, so $E f(x)=P f_{1}(x)=P^{*} \delta_{x}\left(f_{1}\right)=f_{1}(x)=f(x)$. This completes the proof.

REMARK. The extension property fails for $X=\beta N$ and $Z=\beta N \backslash N$ [8, p. 375]. 
4.2 Example. We give an example to show that the hypothesis $L+M=C(X)$ is really needed for Propositions 2.3 and 2.4. Let $X=\{1,2,3,4\}$ with the discrete topology, so that $C(X)$ is essentiallay $R^{4}$. For simplicity we identify $f \in C(X)$ with its values $(a, b, c, d)$. Let

$$
L=\{(a,-a, b, b): a, b \in R\},
$$

so $L^{\perp}$ is the span of the measures whose values at points are $(1,1,0,0)$ and $(0,0,1,-1)$. Now $M=\{(a, a, b, b): a, b \in R\}$ so $M+L \neq C(X) . \quad I=I_{0}=$ $\{(0,0, a, a): a \in R\}$, which is not an ideal. However, $I \subset M$, so 2.4 fails. Further, $m=\left(0,0, \frac{1}{2},-\frac{1}{2}\right)$ is an extreme measure in $\left(L^{\perp}\right)_{1}$, but $m(e)=0$, so 2.3 fails.

We now mention without details some other simple examples we have. (i) $L+M=C(X), I_{0}$ is not an ideal, $I \neq I_{0}, I \not \subset M$; (ii) $L+M=C(X), I_{0}$ is an ideal, $I$ is not; (iii) $L+M \neq C(X), I_{0}$ is an ideal, $I$ is not, and $I \not \subset M$.

5. Remarks. I do not know whether our result is valid in noncommutative $C^{*}$-algebras. It is known that for unital JC algebras, the identity $P(P a P b)=P(a P b)$ holds, where multiplication is the Jordan product [10, Lemma 1.1].

From [2] it is clear that contractive projections are more complicated than Markov projections, and it is not generally true that $(\operatorname{ran} P)(\operatorname{ker} P) \subset \operatorname{ker} P$. In fact, if $f \in C_{C}(X)$ (the complex continuous functions) and $m$ is extreme in $\left(L^{\perp}\right)_{1}$, where $L=\operatorname{ker} P$, then on supp $m, P f$ is a constant times the Radon-Nikodym derivative $d|m| / d m$. (If $P$ is Markov, then $|m|= \pm m$, so $P f$ is constant on supp $m$.) It is an easy consequence of this that $(\operatorname{ran} P)(\operatorname{ran} P)^{-} \subset$ mult $P$, or, equivalently, the identity $P\left(P f(P g)^{-} P h\right)=P\left(f(P g)^{-} P h\right)$ - the bar stands for complex conjugation. In fact, this is proved for general $C^{*}$-algebras in [11, Corollary 3].

Finally, in view of Proposition 4.1, it would be interesting to find characterizations - topological or analytic-of compact spaces for which the extension theorem holds. See [8] for references.

I am grateful to A. Iwanik for pointing out that our result fails if $L$ is not a proper subspace of $C(X)$.

\section{REFERENCES}

1. G. Birkhoff, Moyennes de fonctions borneés, Algèbre et Théorie des Nombres, Colloques Internat. Centre National Recherche Scientifique, no. 24, Paris, France, 1950, pp. 143-153.

2. Y. Friedman and B. Russo, Contractive projections on $C_{0}(K)$, Trans. Amer. Math. Soc. 273 (1982), $57-73$

3. J. Kelley, Averaging projections on $C_{\infty}(X)$, Illinois J. Math. 2 (1958), 214-223.

4. G. Leibowitz, Lectures on complex function algebras, Scott, Foresman, and Co., Glenview, Ill., 1970.

5. S. Lloyd, On continuous projections in spaces of continuous functions, Pacific J. Math. 13 (1963), 171-175.

6. __ A mixing condition for extreme left invariant means, Trans. Amer. Math. Soc. 125 (1966), 461-481.

7. G. Seever, Nonnegative projections on $C_{0}(X)$, Pacific J. Math. 17 (1966), 159-166.

8. Z. Semadeni, Banach spaces of continuous functions, vol. I, PWN, Warsaw, 1971.

9. D. Wulbert, Averaging projections, Illinois J. Math. 13 (1969), 689-693.

10. E. Effros and E. Størmer, Positive projections and Jordan structure in operator algebras, Math. Scand. 45 (1979), 127-138.

11. M. Youngson, Completely contractive projections on $C^{*}$-algebras, Quart. J. Math. Oxford Ser. (2) 34 (1983), 507-511. 